\section{Programa de Triagem Neonatal do Hospital das Clínicas da Faculdade de Medicina de Ribeirão Preto, Universidade de São Paulo, Brasil}

\author{
Neonatal Screening Program at the University \\ Hospital of the Ribeirao Preto School of Medicine, \\ São Paulo University, Brazil
}

\author{
${ }^{1}$ Faculdade de Medicina de \\ Ribeirão Preto, Universidade \\ de São Paulo, Ribeirão Preto, \\ Brasil. \\ Correspondência \\ L. M. Z. Maciel \\ Departamento de Clínica \\ Médica, Faculdade de \\ Medicina de Ribeirão Preto, \\ Universidade de São Paulo. \\ Av. Bandeirantes 3900, \\ Ribeirão Preto, SP \\ 14049-900, Brasil. \\ lmzmacie@fmrp.usp.br
}

\begin{abstract}
The Neonatal Screening Program at the University Hospital of the Ribeirao Preto School of Medicine, São Paulo University, Brazil, was introduced in 1994. As of December 2005, congenital hypothyroidism had been diagnosed in 76 infants, phenylketonuria in 10, and hemoglobinopathies in 25, representing incidence rates of 1:2,595, $1: 19,409$, and 1:4,120, respectively. A total of 2,747 newborns had the sickle cell trait, i.e., were heterozygous for the sickle mutation (1:37.5 live births). The program's mean coverage during this period was $94.5 \%$. There was major improvement in the parameters for evaluating the program's quality, although they were still far from ideal. Public-awareness campaigns on the importance of neonatal screening are needed to increase the program's coverage. Setting postnatal day 3 as the standard Day for the Heel Stick Test would help ensure treatment at earlier ages, thus improving prognosis for affected infants.
\end{abstract}

Neonatal Screening; Congenital Hypothyroidism; Phenylketonuria; Haemoglobinopathies; Health Programs and Plans
Patrícia Künzle Ribeiro Magalhães 1

Marlene de Fátima Turcato 1

Ivan de Lucena Angulo 1

Léa Maria Zanini Maciel 1

\section{Introdução}

Na década de 60, Guthrie \& Susi ${ }^{1}$ demonstraram que a fenilcetonúria poderia ser precocemente detectada logo após o nascimento pela determinação da concentração de fenilalanina em amostras de sangue seco colhido em papel-filtro dos recém-nascidos. Desde então, vários programas de triagem neonatal foram implantados em todo o mundo, tornando-se parte fundamental dos programas de saúde pública.

A maioria desses programas teve início com a triagem da fenilcetonúria (PKU) e do hipotireoidismo congênito, duas doenças com incidência relativamente alta e que, caso não sejam detectadas e tratadas precocemente, levam a retardo mental irreversível. Atualmente, outras doenças foram incluídas no rastreamento dos diferentes programas: doença falciforme, fibrose cística, galactosemia, hiperplasia congênita de adrenal, deficiência de biotinidase, deficiência de glicose 6 fosfato, toxoplasmose e homocistinúria, entre outras.

No Brasil, o Programa de Triagem Neonatal teve início em 1976 com o projeto pioneiro coordenado pelo Prof. Benjamin Schmidt para a triagem da PKU junto à Associação de Pais e Amigos dos Excepcionais de São Paulo (APAE-SP). Somente em 1983, a triagem para hipotireodismo congênito e PKU (teste do pezinho) tornou-se obrigatória no Estado de São Paulo para crianças nascidas em hospitais e maternidades públicos 
(Lei Estadual $n^{\circ}$. 3.914, de 14 de novembro de 1983), sendo que, em 1990, esta obrigatoriedade foi estendida para as crianças nascidas em todo o país, seja na rede pública ou na rede privada ( $L e i$ Federal $n^{\circ}$. 8.069, de 13 de julho de 1990) ${ }^{2}$.

Em 1985, para atender as exigências da lei, o Hospital das Clínicas da Faculdade de Medicina de Ribeirão Preto (HCFMRP) da Universidade de São Paulo firmou convênio com a Escola Paulista de Medicina da Universidade Federal de São Paulo para realização do TSH neonatal e com a APAE-SP para detecção da PKU. Com a montagem dos métodos necessários para a realização dos referidos exames, o HCFMRP efetivamente implantou o seu Programa de Triagem Neonatal para hipotireodismo congênito em 1994 e para PKU em 1995. A partir de agosto de 1996, este programa passou a atender não só crianças nascidas neste hospital, mas todas as crianças nascidas nos 25 municípios que compõem o Departamento Regional de Saúde de Ribeirão Preto, São Paulo (DRS-XIII). Em janeiro de 2002, diante do credenciamento pelo Ministério da Saúde como um dos Serviços de Referência de Triagem Neonatal no Estado de São Paulo (Portaria $n^{o}$. 822, de 06 de junho de 2001) 3, o HCFMRP passou a realizar também o rastreamento de hemoglobinopatias. A partir de novembro de 2002, o Programa de Triagem Neonatal do HCFMRP assumiu a responsabilidade de realizar os testes de triagem neonatal das crianças nascidas nas 22 cidades pertencentes ao DRS de Franca, São Paulo (DRSVIII) e, a partir de fevereiro de 2003, daquelas nascidas nos 19 municípios do DRS de Barretos, São Paulo (DRS-V), abrangendo, assim, um total de 66 municípios.

Para que um Programa de Triagem Neonatal consiga atingir plenamente seus objetivos é fundamental que sejam realizadas avaliações periódicas da qualidade do programa, reportando-se os resultados destas avaliações às autoridades públicas responsáveis, de modo que eventuais modificações possam ser implementadas para aprimorá-lo. Sendo assim, o principal objetivo deste trabalho é avaliar o Programa de Triagem Neonatal do HCFMRP no período de 1994 a 2005, principalmente quanto à abrangência da cobertura, aos tempos decorridos entre o nascimento e a coleta do exame, chegada do exame ao laboratório e liberação dos resultados, bem como à idade das crianças no momento do início do tratamento.

\section{Material e métodos}

Esta é uma avaliação descritiva e retrospectiva, envolvendo a população de crianças rastreadas pelo Programa de Triagem Neonatal do HCFMRP, desde sua implantação em 1994 até dezembro de 2005. Neste período, foram rastreadas 197.265 crianças para hipotireodismo congênito, 194.092 crianças para PKU e 103.021 crianças para hemoglobinopatias.

A coleta do sangue para exame é realizada em papel-filtro (Schleicher \& Schuell 903) por punção do calcanhar das crianças, nos postos de saúde, hospitais e maternidades dos municípios participantes do programa, por membro da equipe de enfermagem ou por técnico devidamente treinado para esta finalidade. Nos 66 municípios participantes do Programa de Triagem Neonatal do HCFMRP estão cadastrados 140 postos de coleta (41 no DRS de Barretos, 36 no DRS de Franca e 63 no DRS de Ribeirão Preto).

As amostras são encaminhadas ao Laboratório de Triagem Neonatal do HCFMRP por motoristas de ambulâncias que conduzem pacientes para consultas médicas no hospital, ou então, por malotes enviados pelos postos de saúde do Município de Ribeirão Preto. Este procedimento foi adotado para reduzir gastos com o programa, considerando-se as proximidades dos municípios e o transporte freqüente de pacientes realizado pelas prefeituras. No laboratório, o material é examinado quanto ao preenchimento dos dados do cartão, qualidade da amostra sangüínea e idade da criança no momento da coleta. Os cartões, nos quais se identifiquem amostras inadequadas ou coletadas em idade inferior a 48 horas, são devolvidos aos respectivos postos de coleta para uma segunda coleta, enquanto os demais são encaminhados para análise.

A triagem para hipotireodismo congênito é realizada pela dosagem de TSH através de método imunofluorimétrico (Kit Neonatal hTSH; DELFIA, Wallac Oy, Finlândia, no período de 1994 a 2002 e AutoDELFIA a partir de2003, Wallac Oy, Turku, Finland). A dosagem de fenilalanina é realizada por método colorimétrico (Kit Quantase; Quantase Ltda., Escócia - de 1994 a 1999 - e NeoLISA PKU Kit; Internacional Científica Ltda., São José dos Campos, Brasil - a partir de 2000) e a triagem de hemoglobinopatias pelo método da cromatografia líquida de alta performance (HPLC) associado à cromatografia de troca catiônica (Programa de Células Falciformes VARIANT; Bio-Rad Laboratories Inc., California, Estados Unidos).

As crianças com valores de TSH neonatal > $10 \mu \mathrm{IU} / \mathrm{mL}$ são convocadas para uma avaliação clínica e confirmação do resultado no soro (dosagem de $\mathrm{TSH}, \mathrm{T}_{4}$ total e $\mathrm{T}_{4}$ livre). Naquelas em que o diagnóstico é confirmado ( $\mathrm{TSH}>10 \mu \mathrm{UI} / \mathrm{mL}$ ), realiza-se cintilografia da tireóide com $99 \mathrm{mTc}$, no intuito de definir a etiologia do hipotireoi- 
dismo, e institui-se o tratamento com levo-tiroxina na dose de 10 a $15 \mu \mathrm{g} / \mathrm{kg} / \mathrm{dia}$. Aquelas com $\mathrm{TSH}$ neonatal entre 5 e $10 \mu \mathrm{IU} / \mathrm{mL}$ têm o exame repetido em papel-filtro e, se mantidos estes valores, são convocadas para um exame clínico e dosagem de $\mathrm{TSH}$ e $\mathrm{T}_{4}$ no soro, cujos resultados vão nortear a conduta: se TSH > $10 \mu \mathrm{IU} / \mathrm{mL}$, o tratamento com levo-tiroxina é iniciado; se $\mathrm{TSH}$ entre 4 e $10 \mu \mathrm{IU} / \mathrm{mL}$, a criança permanece em observação com dosagens hormonais periódicas e, se TSH $<4 \mu \mathrm{IU} / \mathrm{mL}$, ou seja, normal, recebe alta do serviço.

As crianças com valores de fenilalanina $>4 \mathrm{mg} \%$ são convocadas para uma avaliação clínica e coleta de sangue, por punção venosa, para análise de aminoácidos no plasma, incluindo tirosina. As análises são feitas por cromatografia de troca iônica, em parceria com o Centro de Química de Proteínas da FMRP-USP. Sendo confirmados valores de fenilalanina plasmática acima de $10 \mathrm{mg} \%$, com taxas normais de tirosina, o tratamento dietético específico para PKU é iniciado. Os casos em que se constatam valores de fenilalanina entre 4 e $10 \mathrm{mg} \%$ são considerados como hiperfenilalaninemia benigna e continuam em observação, com retornos ambulatoriais e dosagens periódicas de aminoácidos.

Os recém-nascidos com padrão de hemoglobina $\mathrm{HbS}$ ou HbSC, aos dois meses de vida, têm consulta agendada no Pronto Atendimento de Pediatria do HCFMRP, onde são avaliados clinicamente e é iniciada a antibiótico-profilaxia com penicilina. Deste ambulatório, são encaminhados ao Centro Regional de Hemoterapia do HCFMRP, onde a doença falciforme é confirmada por eletroforese de hemoglobina e o seguimento clínico efetuado.

As variáveis estudadas foram: número de crianças nascidas vivas, no período de 1997 a 2005, nos DRSs pertencentes ao Programa de Rastreamento Neonatal do HCFMRP (Ribeirão Preto, Franca e Barretos) segundo o Sistema de Informações sobre Nascidos Vivos (SINASC; Departamento de Informática do SUS. http:// tabnet.datasus.gov.br/cgi/deftohtm.exe?sinasc/ cnv/nvSP.def, acessado em 08/Abr/2008), número de crianças rastreadas pelo programa em cada ano, número de crianças afetadas por doença, tempo decorrido entre o nascimento da criança e a coleta do exame, tempo decorrido entre a coleta do exame e sua chegada ao laboratório, tempo decorrido entre a chegada da amostra ao laboratório e a liberação dos resultados, idade das crianças no momento do início do tratamento.

A partir dos dados individuais dos vários municípios foram calculados média, mediana e desvio-padrão. Para o cálculo da cobertura do programa foi utilizada a fórmula: cobertura = nú- mero de rastreados X 100/número de nascidos vivos. A incidência das doenças foi calculada a partir do número de casos confirmados dividido pelo número de nascidos vivos, no período estudado.

\section{Resultados}

Desde a implantação do Programa de Triagem Neonatal do HCFMRP, em 1994, até dezembro de 2002, o número de crianças rastreadas mantevese mais ou menos constante ao longo dos anos (média 16.651 \pm 768 crianças por ano; mediana = 16.509). A partir de 2003, com a expansão do programa para os DRSs de Franca e Barretos, o número de exames realizados praticamente dobrou, aumentando para $29.649 \pm 768$ crianças por ano (mediana $=29.953$ ).

No período de 1994 a 2005, entre as 197.265 crianças triadas para hipotireodismo congênito, foram encontradas 76 afetadas pela doença e entre as 194.092 crianças rastreadas para PKU, dez apresentaram tal diagnóstico. Com base nestes dados, a incidência de hipotireodismo congênito e de PKU na população estudada foi, respectivamente, 1:2.595 e 1:19.409. Foram ainda diagnosticadas sete crianças com hipertirosinemia transitória. Das 103.021 crianças que realizaram o rastreamento para anemia falciforme e outras hemoglobinopatias, 14 crianças tiveram o diagnóstico de anemia falciforme e 11 de doença SC, estabelecendo-se incidência de 1:7.358 e 1:9.365, respectivamente. Além disso, foram realizados 2.747 diagnósticos de traço falciforme, o que define ocorrência de 1 afetado para cada 37,5 nascimentos (2,6\%) (Tabela 1$)$.

Com base nos dados obtidos do SINASC relativos ao número de crianças nascidas vivas em cada município componente do DRS de Ribeirão Preto nos anos de 1997 a 2005 e dos DRSs de Franca e Barretos nos anos de 2003 a 2005, e o número de crianças rastreadas pelo programa em cada ano, foi possível o cálculo da abrangência do Programa de Triagem Neonatal do HCFMRP. Deve-se, no entanto, ressaltar que $20 \%$ das crianças nascidas no município de Ribeirão Preto não têm o seu exame realizado pelo Sistema Único de Saúde (SUS) e, em parte delas, o teste é encaminhado para outros centros de triagem neonatal ou laboratórios particulares. Sendo assim, para tornar mais fidedigna a informação, os dados relativos ao município de Ribeirão Preto foram excluídos do cálculo da cobertura. A cobertura média do Programa de Triagem Neonatal do HCFMRP foi, respectivamente, 93,3\%, 99\% e 93,7\% nos DRSs de Ribeirão Preto, Franca e Barretos (média geral $=94,5 \%$ ) (Tabela 2 ) . 
Incidência de hipotireoidismo congênito, fenilcetonúria e doença falciforme na população rastreada pelo Programa de Triagem Neonatal do Hospital das Clínicas da Faculdade de Medicina de Ribeirão Preto (HCFMRP) da Universidade de São Paulo, Brasil, no período de 1994 a 2005.

\begin{tabular}{lccc}
\hline Doenças & $\begin{array}{c}\text { Número total de } \\
\text { crianças rastreadas }\end{array}$ & $\begin{array}{c}\text { Número total de } \\
\text { crianças afetadas }\end{array}$ & $\begin{array}{c}\text { Incidência da doença } \\
\text { na nossa população }\end{array}$ \\
\hline Hipotireoidismo congênito & 197.265 & 76 & $1: 2.595$ \\
Fenilcetonúria & 194.092 & 10 & $1: 19.409$ \\
Anemia falciforme & 103.021 & 14 & $1: 7.358$ \\
Doença SC & 103.021 & 11 & $1: 9.365$ \\
\hline
\end{tabular}

Tabela 2

Cobertura do Programa de Triagem Neonatal do Hospital das Clínicas da Faculdade de Medicina de Ribeirão Preto (HCFMRP) da Universidade de São Paulo, Brasil, no período de 1997 a 2005.

\begin{tabular}{lcccc}
\hline Ano & & \multicolumn{2}{c}{ Cobertura (\%) } & \\
& & DRS & Geral * \\
& Ribeirão Preto * & Franca & & \\
\hline 1997 & 90,9 & - & - & - \\
1998 & 91,0 & - & - & - \\
1999 & 94,8 & - & - & - \\
2000 & 95,3 & - & - & - \\
2001 & 93,7 & - & - & - \\
2002 & 94,3 & - & 101,5 & \\
2003 & 93,6 & 98,5 & 89,9 & 97,9 \\
2004 & 92,6 & 100,4 & 89,6 & 94,3 \\
2005 & 93,7 & 98,2 & 93,7 & 93,8 \\
Geral & 93,3 & 99,0 & & - \\
\hline
\end{tabular}

* Excluídos os dados referentes ao Município de Ribeirão Preto.

DRS: Departamento Regional de Saúde.

Os dados relativos aos intervalos de tempo decorridos entre o nascimento da criança e a coleta do exame, entre a coleta do exame e sua chegada ao laboratório e entre a chegada da amostra ao laboratório e a liberação dos resultados são apresentados na Figura 1. A idade de coleta do teste de triagem neonatal, que em 1996 era em torno de 17 dias, diminuiu progressivamente até em torno de 8 dias em 2003 e, a partir daí, não mais se modificou. Quanto ao número de dias entre a coleta do exame e sua chegada ao laboratório, houve uma diminuição importante entre 1996 e 1997, mas posteriormente manteve-se constante em torno de 5 a 6 dias, até com discreto aumento no último semestre de 2005. O tempo decorrido entre a chegada do exame ao laboratório e a liberação do resultado também não se modificou ao longo dos anos, estando sempre próximo de 2 a 3 dias.

No ano de $2002,50 \%$ a $60 \%$ dos exames eram colhidos na primeira semana de vida do recémnascido e esta porcentagem de coleta aumentou para em torno de $70 \%$ no ano de 2005.

O tempo decorrido entre a convocação das crianças, cujo TSH neonatal encontrava-se alterado, e sua primeira avaliação no nosso serviço foi, em média, de um dia. A idade média de início de tratamento das crianças, cujo diagnóstico de hipotireodismo congênito foi confirmado, foi de $25,9 \pm 14,2$ dias de vida $(\mathrm{N}=60$; mediana $=22,5$ dias; range $=10-86$ ). Deste cálculo foram excluídas as crianças com elevações tardias do TSH e aquelas submetidas à transfusão sangüínea, cujo diagnóstico e início do tratamento foram obvia- 
Figura 1

Idade da criança por ocasião da coleta do teste de triagem neonatal (1a), tempo entre a data de coleta do teste e a sua chegada ao laboratório (1b) e tempo entre a chegada do exame ao laboratório e a liberação do resultado (1c), no Programa de Triagem Neonatal do Hospital das Clínicas da Faculdade de Medicina de Ribeirão Preto (HCFMRP) da Universidade de São Paulo, Brasil.

1a)

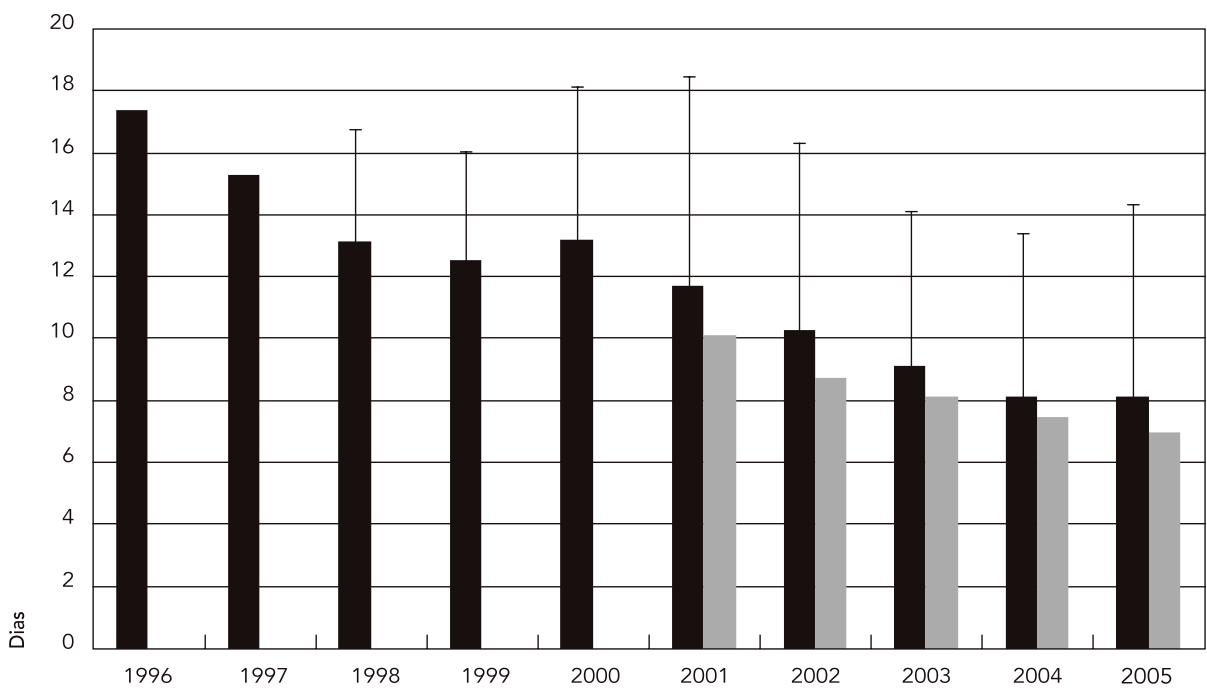

1b)

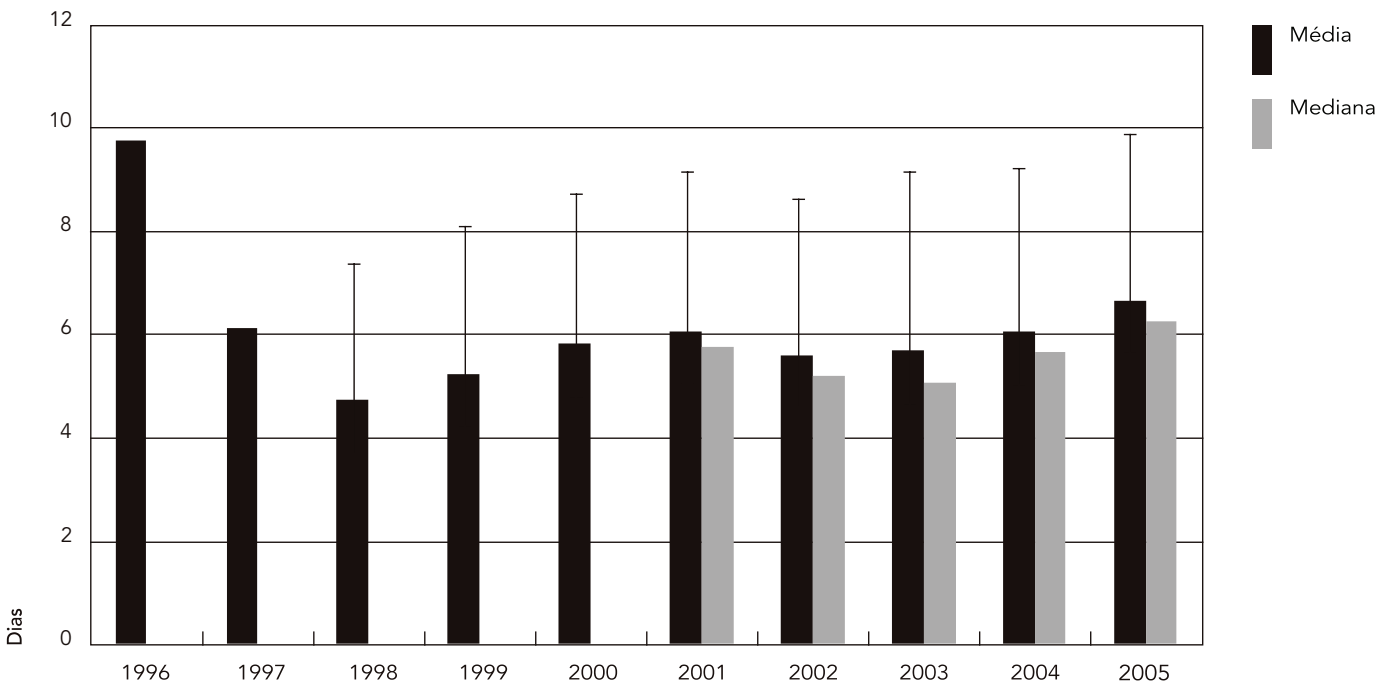

(continua) 
1c)

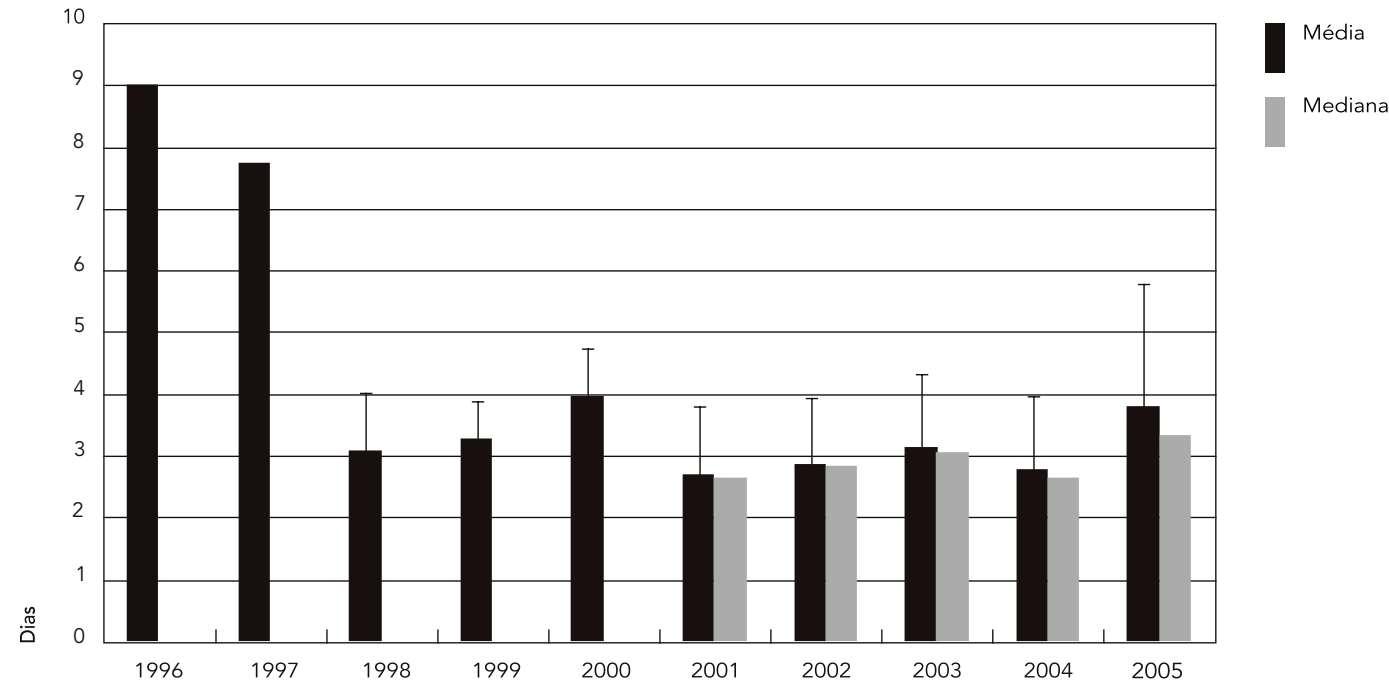

mente retardados. Comparando-se estes valores com aqueles encontrados antes da implantação efetiva do Laboratório de Triagem Neonatal no HCFMRP, quando os exames eram realizados em parceria com a APAE-SP, houve uma grande diminuição na idade de início de tratamento das crianças, pois naquela época elas apresentavam, em média, 70,7 \pm 40,7 dias de vida $(\mathrm{N}=15$; mediana $=61$ dias; range $=13-180$ ) (Figura 2 ).

Com relação à fenilcetonúria clássica, a média de idade das crianças ao diagnóstico foi de $25,7 \pm 17,5$ dias de vida $(\mathrm{N}=10$; mediana $=19,5$ dias; range $=9-60$ ).

\section{Discussão}

O HCFMRP é um dos sete Centros de Referência de Triagem Neonatal do Estado de São Paulo, estando credenciado pelo Ministério da Saúde na fase II, o que torna obrigatória a triagem neonatal do hipotireodismo congênito, da PKU e das hemoglobinopatias. As duas primeiras doenças devem ser diagnosticadas e tratadas o mais precocemente possível para evitar seqüelas neurológicas graves para o paciente, enquanto as hemoglobinopatias são doenças que cursam com elevada morbidade, a qual pode ser amenizada empregando-se alguns cuidados básicos de saúde (profilaxia contra infecções com penicilina, uso de ácido fólico, vacinações).
O método diagnóstico empregado para triagem neonatal de hipotireodismo congênito apresenta sensibilidade e especificidade de $97 \%$ e 99\%, respectivamente, enquanto estas duas propriedades giram em torno de $99 \%$ para o rastreamento de PKU 4,5. Desta forma, a triagem neonatal apresenta valor inquestionável como política de saúde pública, sendo obrigatória na maioria dos países 5,6,7. No entanto, para alcançar seu principal objetivo, que é estabelecer o diagnóstico e iniciar o tratamento destas doenças o mais precocemente possível, é importante que existam padrões de tempo para cada etapa do processo de rastreamento, uma vez que atrasos em uma ou mais etapas levarão a retardos inaceitáveis no tratamento das crianças afetadas $8,9,10,11,12,13,14$

A idade ideal para a coleta da primeira amostra de sangue para o rastreamento com TSH é do 3 o ao 5 o dia de vida, quando já ocorreu a diminuição do pico pós-natal de elevação fisiológica do TSH 8,11,12. Para a triagem da PKU, também se deve esperar pelo menos 48 horas de vida da criança, quando ela já recebeu leite suficiente para que haja acúmulo de fenilalanina no sangue, caso seja afetada 15. Em nosso programa, a idade média de coleta do exame melhorou muito desde o início do programa, diminuindo de 17 dias para oito dias, o que deve refletir o empenho do Ministério da Saúde, Secretarias Municipais de Saúde e Centros de Triagem para a divulgação do 
Idade média do início do tratamento das crianças com hipotireoidismo congênito no Programa de Triagem Neonatal do Hospital das Clínicas da Faculdade de Medicina de Ribeirão Preto (HCFMRP) da Universidade de São Paulo, Brasil.

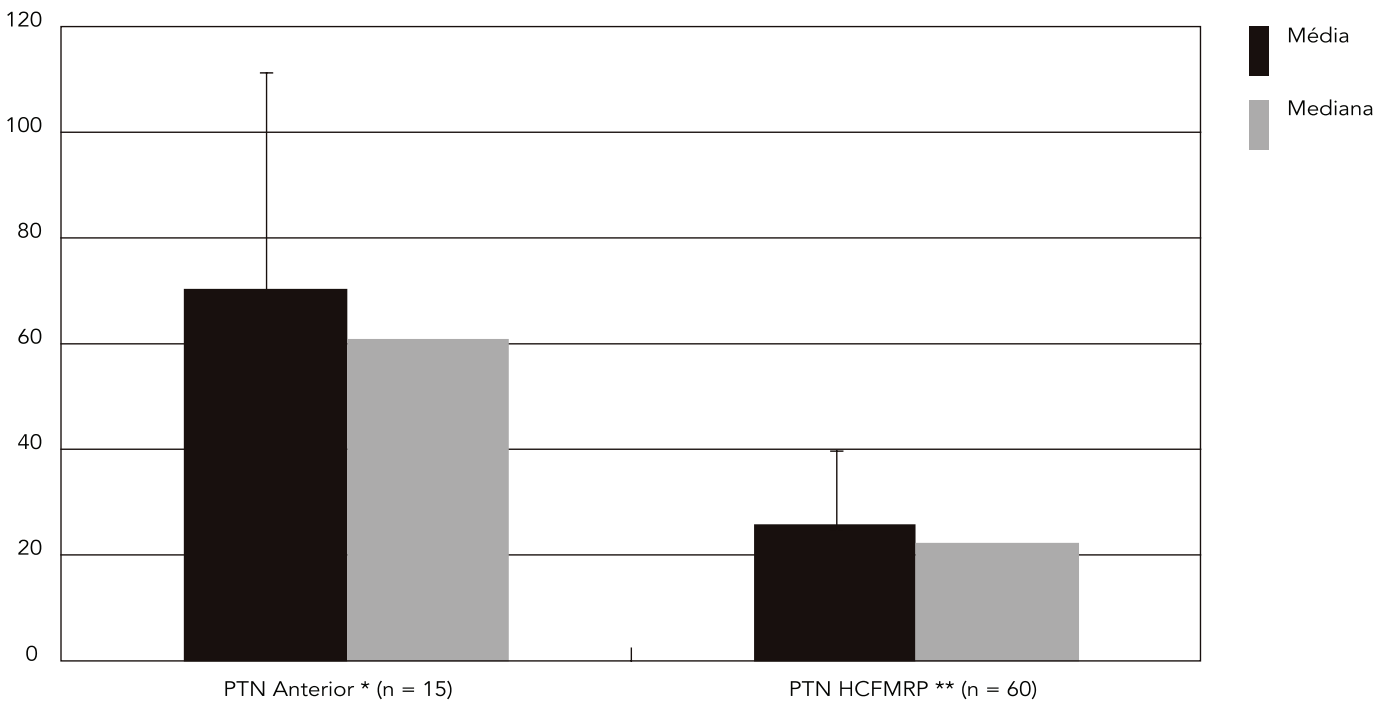

teste de triagem neonatal e orientação quanto à importância da sua realização. Este valor encontra-se próximo dos limites estabelecidos como ideais na Portaria $n^{o} .822^{3}$ do Ministério da Saúde (3 a 7 dias de vida) e inferior à idade de coleta do teste de triagem neonatal em outros estados brasileiros: 11 a 12 dias no Paraná 16, 12 dias no Sergipe 17,18, 17 dias em Santa Catarina 19 e 29,8 dias na Paraíba 20. Deve-se, no entanto, enfatizar que ainda é baixa a porcentagem de crianças que colhem o exame na primeira semana de vida na nossa região (em torno de $70 \%$, quando o ideal seria a totalidade delas). Em uma análise recente da triagem do hipotireodismo congênito na Escócia 21 , a mediana da idade de coleta do teste de triagem neonatal foi de seis dias, sendo que $93,8 \%$ dos exames foram colhidos antes dos oito dias de vida.

No Programa de Triagem Neonatal do HCFMRP recomenda-se que, após a coleta, as amostras sejam enviadas ao laboratório o mais breve possível, ou seja, assim que estiverem secas. Entretanto, como alguns postos de coleta aguardam que se acumulem amostras para depois enviá-las para análise, o tempo médio decorrido entre a coleta do exame e sua chegada ao laboratório gira em torno de uma semana, maior que o recomendado pelo Ministério da Saúde 3, que é de até cinco dias úteis, proporcionando atraso adicional para o diagnóstico e tratamento. Este atraso no tempo gasto para a chegada da amostra ao laboratório também ocorre em outros estados, tais como Distrito Federal 22, Paraná 16, Santa Catarina 19, Sergipe 17,18 e Paraíba 20, possivelmente pelo mesmo motivo.

O tempo decorrido entre a chegada do exame ao laboratório e a liberação do resultado vem se mantendo em torno de três dias ao longo dos anos, valor menor do que aquele recomendado pelo Ministério da Saúde ${ }^{3}$ que é de cinco dias úteis, bastante inferior à media nacional que é de dez dias 23, mas semelhante ao encontrado em Santa Catarina 19 e na área de Bath (Inglaterra) 14

A regionalização do Programa de Triagem Neonatal proporcionada pela implantação do Laboratório de Triagem Neonatal no HCFMRP permitiu que o início do tratamento das crianças afetadas para hipotireodismo congênito diminuísse de 70,7 \pm 40,7 dias de vida para 25,9 \pm 14,2 dias, ocorrendo em grande parte das vezes dentro do tempo considerado ideal, ou seja, até a $2^{\text {a }}$ semana de vida 9,10,24,25,26,27. Na avaliação realizada em Santa Catarina, em 1998, o percentual de crianças atendidas até 28 dias de vida ainda foi de 46,7\% 19 e, no Paraná, a idade média de início de tratamento em 1996 foi de 25,8 dias 16, bastante semelhante a nossa. Dados nacionais 
obtidos pela Sociedade Brasileira de Triagem Neonatal nos anos de 2002 e 2003 mostram que o tratamento do hipotireodismo congênito se dá em uma idade média de $34 \pm 17$ dias ${ }^{23}$. No estudo escocês ${ }^{21}$, a mediana de idade de início de tratamento foi de 11 dias, sendo que $12 \%$ das crianças avaliadas tiveram um início de tratamento considerado tardio ( $>16$ dias de vida).

Um parâmetro de importância crucial na avaliação de um Programa de Triagem Neonatal é a sua cobertura. Em nosso serviço, no período de 1994 a 2005, a cobertura geral foi de 94,5\%, inferior àquela encontrada em países desenvolvidos: $100 \%$ na Itália 28, 99,95\% na Escócia 21, 95\% na Nova Zelândia 29, 99\% na Austrália 30 e 98,6\% na Califórnia 31, e aquém do objetivo de qualquer Programa de Triagem Neonatal, que é o rastreamento de $100 \%$ dos nascidos vivos ${ }^{13}$, porém bem melhor do que a média de cobertura nacional que, em 2005, foi de $80,2 \% 2$. Deve-se ressaltar que, no Brasil, a taxa de cobertura é bastante variável, com estados como Santa Catarina, que apresentou uma cobertura de 91,4\% em 1997 19, estados com coberturas muito baixas, como Paraíba que rastreou em 2001 e 2002 apenas 32,2\% de seus nascidos vivos 20 e outros, como Sergipe, com coberturas intermediárias $(71,5 \%) 17$. Na América Latina, a cobertura da triagem neonatal também é heterogênea. Em países como Cuba, Costa Rica, Uruguai e Chile, que apresentam um Programa de Triagem Neonatal mais desenvolvido, a cobertura chega a próximo de $100 \%$; outros países estão em fase de implantação do Programa de Triagem Neonatal e apresentam coberturas desde $4 \%$ a $6 \%$ (Nicarágua e Peru) até $60 \%$ a $80 \%$ (México e Argentina). Paralelamente, há países, como El Salvador, Haiti e Honduras, onde o Programa de Triagem Neonatal virtualmente não existe 32 .

Finalizando, para que um Programa de Triagem Neonatal consiga atingir plenamente os seus objetivos, é essencial que algumas metas sejam cumpridas: (1) coleta da amostra san güínea obtida corretamente e em tempo adequado; (2) encaminhamento rápido da amostra ao laboratório de referência; (3) realização dos exames pelo laboratório obedecendo a rigoroso controle de qualidade; (4) rápida comunicação dos resultados dos exames; (5) centro de referência dotado de médico(s) treinado(s) para estabelecer(em) o diagnóstico preciso e de estrutura para o seguimento clínico das crianças afetadas; (6) avaliações periódicas da qualidade do programa, reportando-se os resultados destas avaliações às autoridades responsáveis, a fim de que eventuais melhorias possam ser implementadas. A análise global de todos os parâmetros avaliados evidencia que o Programa de Triagem Neonatal do HCFMRP, desde sua implantação, tenta cumprir da melhor maneira possível esses objetivos, apresentando, de maneira geral, um bom desempenho, em muitos pontos similares aos referidos como padrão. Vários aspectos, porém, podem e devem ser otimizados, tais como ampliação da taxa de cobertura e diminuição da idade da coleta do exame, bem como do tempo transcorrido entre a coleta e a chegada da amostra ao laboratório. Esta melhora, no entanto, não depende apenas do laboratório, onde são realizados os exames, e/ou do ambulatório, onde são acompanhados os pacientes afetados, mas de todo um conjunto de pessoas e instituições, que engloba desde os responsáveis pela criança (mãe e/ou pai), hospital ou maternidade onde nasceu a criança, postos responsáveis pela coleta adequada das amostras e seu envio de maneira ágil, Secretarias de Saúde dos municípios (realização de campanhas de esclarecimento quanto à importância do teste) até o Serviço de Referência de Triagem Neonatal. Semelhante ao já instituído na França 33, a adoção do 3o dia de vida do recém-nascido como sendo o "Dia da Triagem Neonatal”, associada à divulgação abrangente, poderiam representar uma estratégia para ampliar a atenção neste programa de fundamental importância para a Saúde Pública. Com isto será possível ampliar a cobertura e encurtar o tempo para o início do tratamento nas crianças afetadas, proporcionando, assim, melhores perspectivas para os afetados. 


\section{Resumo}

O Programa de Triagem Neonatal do Hospital das Clínicas da Faculdade de Medicina de Ribeirão Preto, Universidade de São Paulo, Brasil, instituído em 1994 diagnosticou, até 2005, 76 crianças com hipotireoidismo congênito, 10 com fenilcetonúria e $25 \mathrm{com}$ hemoglobinopatias, o que representou uma incidência de 1:2.595, 1:19.409, 1:4.120, respectivamente. Foram diagnosticadas 2.747 crianças com traço falciforme (1:37,5 nascidos vivos). A cobertura média do programa foi de 94,5\%. Houve uma considerável melhora nos parâmetros de avaliação da qualidade do programa no período, porém, sem atingir os índices ideais. Campanhas visando à maior divulgação da importância da triagem neonatal são necessárias para aumentar a cobertura e a instituição do 3o dia de vida do recémnascido como sendo o Dia do Teste do Pezinho poderia contribuir para que idades mais precoces de tratamento fossem atingidas, melhorando o prognóstico das crianças acometidas.

Triagem Neonatal; Hipotireoidismo Congênito; Fenilcetonúria; Hemoglobinopatias; Planos de Programas de Saúde

\section{Colaboradores}

P. K. R. Magalhães e L. M. Z. Maciel foram responsáveis pela revisão bibliográfica, análise dos dados, estruturação e redação do artigo. M. F. Turcato e I. L. Angulo participaram da análise dos dados, da discussão dos resultados e da revisão do artigo.

\section{Agradecimentos}

Os autores agradecem à Lucia Helena Carlucci, Giselle Aparecida Caixe de Carvalho Paixão e Léia Silva de Souza, técnicas do Laboratório de Triagem Neonatal; Beatriz Bartholomeu Franco, assistente social do programa e Maria Luísa Barato de Sousa e Andréa Pimentel Alvim pelos serviços de secretariado.

\section{Referências}

1. Guthrie R, Susi A. A simple phenylalanine method for detecting phenylketonuria in large populations of newborn infants. Pediatrics 1963; 32:338-43.

2. Carvalho TM, Santos HP, Santos IC, Vargas PR, Pedrosa J. Newborn screening: a national public health programme in Brazil. J Inherit Metab Dis 2007; 30:615.

3. Ministério da Saúde. Portaria $n^{\circ}$. 822, de 06 de junho de 2001. http://dtr2001.saude.gov.br/sas/ PORTARIAS/Port2001/GM/GM-822.htm (acessado em 19/Nov/2008).

4. Pharoah PO, Madden MP. Audit of screening for congenital hypothyroidism. Arch Dis Child 1992; 67:1073-6.

5. Pollitt RJ, Green A, McCabe CJ, Booth A, Cooper NJ, Leonard JV, et al. Neonatal screening for inborn errors of metabolism: cost, yield and outcome. Health Technol Assess 1997; 1:i-iv, 1-202.

6. LaFranchi S. Detecção neonatal de hipotireoidismo congênito: uma história de sucesso? Arq Bras Endocrinol Metab 1995; 39:80-8.
7. Loeber JG. Neonatal screening in Europe; the situation in 2004. J Inherit Metab Dis 2007; 30:430-8.

8. American Academy of Pediatrics AAP Section on Endocrinology and Committee on Genetics, American Thyroid Association Committee on Public Health. Newborn screening for congenital hypothyroidism: recommended guidelines. Pediatrics. 1993; 91:1203-9.

9. American Academy of Pediatrics, Rose SR; Section on Endocrinology and Committee on Genetics, American Thyroid Association, Brown RS; Public Health Committee, Lawson Wilkins Pediatric Endocrine Society, et al. Update of newborn screening and therapy for congenital hypothyroidism. Pediatrics 2006; 117:2290-303.

10. Bongers-Schokking JJ, Koot HM, Wiersma D, Verkerk PH, Muinck Keizer-Schrama SM. Influence of timing and dose of thyroid hormone replacement on development in infants with congenital hypothyroidism. J Pediatr 2000; 136:292-7. 
11. Fisher DA. Clinical review 19: management of congenital hypothyroidism. J Clin Endocrinol Metab 1991; 72:523-9.

12. Fisher DA. Physiological variations in thyroid hormones: physiological and pathophysiological considerations. Clin Chem 1996; 42:135-9.

13. Grüters A, Delange F, Giovannelli G, Klett M, Rochiccioli P, Torresani T, et al. Guidelines for neonatal screening programs for congenital hypothyroidism. European Society for Pediatric Endocrinology Working Group on Congenital Hypothyroidism. Horm Res 1994; 41:1-2.

14. Simpson N, Randall R, Lenton S, Walker S. Audit of neonatal screening programme for phenylketonuria and congenital hypothyroidism. Arch Dis Child Fetal Neonatal Ed 1997; 77:F228-34.

15. Pass KA, Lane PA, Fernhoff PM, Hinton CF, Panny SR, Parks JS, et al. US newborn screening system guidelines II: follow-up of children, diagnosis, management, and evaluation. Statement of the Council of Regional Networks for Genetic Services (CORN). J Pediatr 2000; 137(4 Suppl):S1-46.

16. França SN. Análise clínico-laboratorial dos pacientes com hipotireoidismo congênito diagnosticado pelo programa de rastreamento neonatal no Estado do Paraná [Tese de Doutorado]. Curitiba: Universidade Federal do Paraná; 1997.

17. Ramalho RJR, Ramalho ARO, Oliveira CRP, AguiarOliveira MH. Evolução do programa de triagem neonatal para o hipotireoidismo congênito e fenilcetonúria no Estado de Sergipe de 1995 a 2003. Arq Bras Endocrinol Metab 2004; 48:890-6.

18. Ramalho RJR, Valido DP, Aguiar-Oliveira MH. Avaliação do programa de triagem para o hipotireoidismo congênito no estado de Sergipe. Arq Bras Endocrinol Metab, 2000; 44:157-61.

19. Nascimento ML, Pires MMS, Nassar SM, Ruhland L. Avaliação do programa de rastreamento neonatal para hipotireoidismo congênito da Secretaria de Estado da Saúde de Santa Catarina. Arq Bras Endocrinol Metab, 2003; 47:75-81.

20. Ramos AJS, Rocha AM, Costa ADM, Benicio AVL, Ramos ALC, Silva CRA, et al. Avaliação do programa de rastreamento de doenças congênitas em Campina Grande-PB, Brasil. Arq Bras Endocrinol Metab2003; 47:280-4.

21. Jones JH, Mackenzie J, Croft GA, Beaton S, Young $\mathrm{D}$, Donaldson MD. Improvement in screening performance and diagnosis of congenital hypothyroidism in Scotland 1979-2003. Arch Dis Child 2006; 91:680-5
22. Franco DB, Maciel RMB, Matsumura LK, Kuni IS, Furuzawa GK, Faria AM, et al. Implantação do programa de rastreamento do hipotireoidismo congênito na Fundação Hospitalar do Distrito Federal: metodologia, resultados, dificuldades e propostas: estudo comparativo com recém-natos de outros estados. Arq Bras Endocrinol Metab 1997; 41:6-13.

23. Sociedade Brasileira de Triagem Neonatal. Dados estatísticos 2002/2003. http://www.sbtn.org.br (acessado em 08/Abr/2008).

24. Dubuis JM, Glorieux J, Richer F, Deal CL, Dussault JH, van Vliet G. Outcome of severe congenital hypothyroidism: closing the developmental gap with early high dose levothyroxine treatment. J Clin Endocrinol Metab 1996; 81:222-7.

25. Fisher DA. The importance of early management in optimizing IQ in infants with congenital hypothyroidism. J Pediatr 2000; 136:273-4.

26. Toublanc JE. Guidelines for neonatal screening programs for congenital hypothyroidism. Working Group for Neonatal Screening in Paediatric Endocrinology of the European Society for Paediatric Endocrinology. Acta Paediatr Suppl 1999; 88:13-4.

27. Revised guidelines for neonatal screening programmes for primary congenital hypothyroidism. Working Group on Neonatal Screening of the European Society for Paediatric Endocrinology. Horm Res 1999; 52:49-52.

28. Olivieri A, Fazzini C, Grandolfo ME, Medda E, Stazi MA, D'Archivio M, et al. La rivalutazione della diagnosi nel'ipotiroidismo congenito. Ann Ist Super Sanita 1999; 35:273-82.

29. Webster D, Essex C. Neonatal screening in New Zealand. BMJ 1996; 312:312.

30. Kurinczuk JJ, Bower C, Lewis B, Byrne G. Congenital hypothyroidism in Western Australia 1981-1998. J Paediatr Child Health 2002; 38:187-91.

31. Waller DK, Anderson JL, Lorey F, Cunningham GC. Risk factors for congenital hypothyroidism: an investigation of infant's birth weight, ethnicity, and gender in California, 1990-1998. Teratology 2000; 62:36-41.

32. Borrajo GJ. Newborn screening in Latin America at the beginning of the 21st century. J Inherit Metab Dis 2007; 30:466-81.

33. Farriaux JP, Vidailhet M, Briard ML, Belot V, Dhondt JL. Neonatal screening for cystic fibrosis: France rises to the challenge. J Inherit Metab Dis 2003; 26:729-44.

Recebido em 09/Abr/2008

Versão final reapresentada em 14/Ago/2008

Aprovado em 29/Ago/2008 